\title{
DYNAMIC STABILITY ANALYSIS WITH NONLOCAL DAMAGE
}

\author{
Gilles Pijaudier-Cabot $\dagger$ and Zdeněk P. Bažant \\ Center for Concrete and Geomaterials, Northwestern University, Evanston, IL 60208, U.S.A.
}

(Received 13 August 1987)

\begin{abstract}
The classic localization instability analysis for strain-softening materials is expanded to dynamic solutions. The nonlocal continuum with local strain, which ensures proper convergence of finite element calculations and physically realistic solutions, is adopted in its simplified form, the nonlocal damage model. The dynamic response of a one-dimensional bar initially in a uniform strain-softening equilibrium state is calculated by finite elements. The stability limit of the bar subjected to a small initial disturbance is computed from the time evolution of the energy dissipation due to damage. The limits found for various lengths of bar are very close to static analytic calculations and exhibit the correct size effect when bars of increasing length are considered.
\end{abstract}

\section{INTRODUCTION}

Since the idea of nonlocal damage was first proposed for strain-softening materials, it has been demonstrated that the energy dissipation cannot localize into a region of vanishing size and that, likewise, static strain localization instability does not permit localization of strains to a Dirac delta function. This result could not be achieved by either stress-displacement constitutive laws [1] or the usual local stressstrain constitutive relations [2]. The conditions for static localization instability were formulated and the influence of various parameters on static localization solutions was studied.

In the numerical examples presented here, we explore the dynamic response of the continuum with nonlocal damage, which was already to some extent discussed in the original presentation of the model [3]. The stability limits for dynamic problems are numerically obtained by finite element analysis and compared to the static localization instability result, which may be regarded as a limiting case of the present dynamic calculations.

\section{NONLOCAL MODEL}

The nonlocal continuum is a continuum in which some state variables are defined by spatial averaging. One constitutive model which is suited for this purpose is the continuum damage theory. In our onedimensional analysis we choose the simplest model in which 'damage is represented by a scalar parameter which affects the secant stiffness of the material. For the present study, this simplified formulation is acceptable. However the extension of this analysis to two or three dimensions would require a more realistic, anisotropic, damage model. For example in the

† On leave from Laboratoire de Mécanique et Technologie, Cachan, France. context of the continuum damage model proposed by Ladevèze [4], and applied to concrete by Mazars and Pijaudier-Cabot [5] such a generalization could be easily made.

The nonlocal damage model is obtained by replacing the local damage $\omega$, by a nonlocal damage scalar denoted $\boldsymbol{\Omega}$ in the constitutive equations. Presented by Pijaudier-Cabot and Bažant [3] and extended later to the concept of nonlocal continuum with local strain [6], the model may be summarized as follows.

The one dimensional stress-strain relations are:

$$
\begin{aligned}
\sigma & =(1-\Omega) \sigma^{\prime}, \\
\sigma^{\prime} & =E \epsilon,
\end{aligned}
$$

where $\sigma$ and $\epsilon$ are the macroscopic stress and strain, $\sigma^{\prime}$ refers to the true stress in the damaged materials defined, e.g., by Lemaitre and Chaboche [7] and $E$ is the Young's modulus of the undamaged material. From thermodynamic considerations [7] the energy dissipation rate due to damage is expressed as

$$
\dot{\Phi}=Y \dot{\Omega}
$$

where $Y$ is called the damage energy release rate;

$$
Y=\frac{1}{2} E \epsilon^{2}
$$

Inspired by the local damage models [5], we assume the nonlocal damage to be defined as a function of the average damage energy release rate $\bar{Y}$, i.e. $\boldsymbol{\Omega}=f(\bar{Y})$, in which

$$
\begin{aligned}
& Y(x)=\frac{1}{V_{r}(x)} \int_{V} \alpha(s-x) Y(s) \mathrm{d} s, \\
& V_{r}(x)=\int_{V} \alpha(s-x) \mathrm{d} s,
\end{aligned}
$$


$\alpha$ is a given weighting function and $V_{r}(x)$ is called the representative volume. A function $f(\bar{Y})$, integrated from experimental data by Zaborski [8], is used in the computations. Along with the loading criterion, this relation is defined as:

$$
\begin{aligned}
& \text { If } F(\bar{Y})=0 \text { and } \dot{F}(\bar{Y})=0 \text { then } \Omega=\omega \\
& \text { If } F(\bar{Y})<0 \text { or if } F(\bar{Y})=0 \text { and } \\
& \dot{F}(\bar{Y})<0 \text { then } \Omega=0,
\end{aligned}
$$

where

$$
\bar{\omega}=1-\left\{\frac{1}{1+b\left(\bar{Y}-Y_{1}\right)^{n}}\right\},
$$

$b, n, Y_{1}$ are empirical constants, $n>1, Y_{1}=$ damage threshold and $F(\bar{Y})=$ empirical loading function, taken as

$$
F(\bar{Y})=\bar{Y}-\kappa(\bar{Y})
$$

$\kappa(\bar{Y})$ is the hardening-softening parameter whose initial value is $Y_{1}$ and afterwards $\kappa(\bar{Y})$ represents the largest value of the average damage energy release rate $\bar{Y}$ reached up to the current time.

In one dimension, the representative volume $V_{r}(x)$ reduces to the characteristic length $l$. We use a uniform weighting function:

$$
\alpha=1 \text { if } s \in[x-l / 2, x+l / 2]
$$

otherwise $\alpha=0$.

The average of $Y(x)$ becomes:

$$
P(x)=\frac{E}{2} \int_{x-1 / 2}^{x+1 / 2} \epsilon^{2}(s) d s .
$$

At the boundary, the domain of length $l$ centered at point $x$ protrudes outside the bar. This protruding part is deleted from the averaging domain and $\bar{Y}$ is computed from the remaining part of the representative volume.

As already demonstrated, this model serves as an effective localization limiter and yields physically realistic solutions with finite energy dissipation at failure and no spurious mesh sensitivity $[6,9]$.

\section{DYNAMIC RESPONSE AND ITS STABILITY}

In a preceding study [6] a uniaxially stressed bar loaded through a spring was considered as a typical problem which reveals the salient characteristics of the nonlocal damage. We now analyze the same bar dynamically, and we will particularly examine stability of the dynamic response. The analysis of the stability of equilibrium of the bar carried out by Bažant [10], Bažant et al.[11] and Bažant and
Zubelewicz [12], and thermodynamically reexamined by Ottosen [13], may be regarded as a limiting case of dynamic stability analysis in which the inertial forces are neglected. In static analysis, the location of the softening zone within the bar is in fact non-unique (and is in reality determined by the statistical variability of the material properties causing one zone to be weaker than another). By contrast, due to inertial forces, this non-uniqueness may be expected to disappear in the dynamic setting.

We consider a bar of length $L$ which is initially in equilibrium under uniformly distributed axial stress $\sigma_{0}$ and strain $\epsilon_{0}$ that is in the softening range. Beginning with time $t=0$, the end points of the bar $(x=0$, $x=L$ ) are forced to displace outwards at a constant velocity $c$ during a short time interval $\delta t$, after which the displacement at the end points remains constant. In the case of elastic behavior this would produce during $\delta t$ a step wave of strain with magnitude $\epsilon=c / v$, where $v=$ elastic wave speed. The response, however, is very different since the initial strain is in the strain-softening range. In a local strain-softening material, the disturbance caused at the boundary would not propagate, and a strain-softening increase of strain would remain limited to the end points. similar to the solution of Bažant and Belytschko [14] as well as the phenomenon of deformation trapping analyzed by $\mathrm{Wu}$ and Freund [15]. For the present nonlocal approach, the disturbance must nevertheless be expected to spread at least over a finite distance into the bar. This question has been analyzed numerically by finite elements with the principal objective of determining the conditions of stability of the dynamic response. The response is considered stable if very small boundary velocities cause a very small change in the state of the bar, and unstable if they cause a finite change in the state of the bar, as measured in terms of displacements, dissipated energy, strains or damage.

The bar is subdivided into $N$ finite elements and the explicil step-by-step time integration procedure, of the same type as used and described in [16]. is used. The material data are selected from the experimental results [8]. As shown in Fig. 1, the relationship of stress versus mean-strain is quite

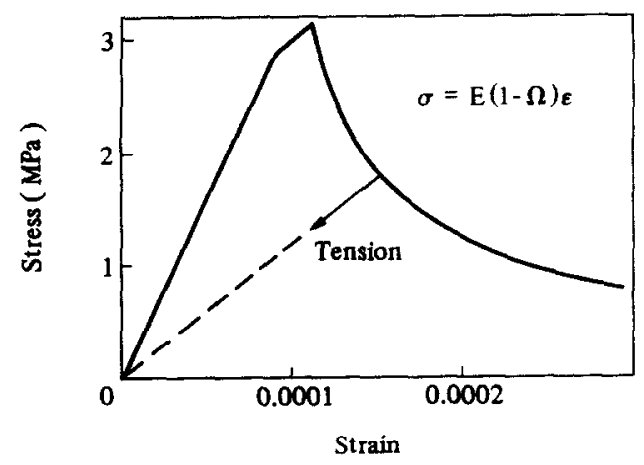

Fig. 1. Stress versus mean-strain curve in tension. 
realistic for concrete or cement paste in tension; elastic modulus $E=32,000 \mathrm{MPa}$, damage threshold $Y_{1}=8540 \times 10^{-6} \mathrm{MPa}$, softening parameters $b=9.7 \times 10^{3}, \quad n=1$, mass density $\rho=0.25 \times 10^{-5} \mathrm{~kg} / \mathrm{mm}^{3}$. The bar length $L$ ranges from $5 l$ to $30 l$, where for convenience the characteristic length of the material is $l=1 \mathrm{~mm}$. The time step is $\Delta t=0.8 \times 10^{-6} \mathrm{sec}$, and the number of elements, $N$, is 75 . The magnitude of the imposed boundary velocity $v$ is chosen such that it initially causes the strain $\epsilon_{0}$ to increase by $0.01 \%$ of $\epsilon_{0}$.

Figure 2 shows the evolution of the axial displacement $u$ at the point $x=L / 4$ for a bar of length $L=5 l$ and for various magnitudes of the initial uniform strain $\epsilon_{0}$, all of them being in the strain-softening range. We see from the figure that for initial strain values $\epsilon_{0}<2.6 \times 10^{-3}$ the histories of displacement as well as strain remain bounded and the strain increments never exceed $0.0001 \epsilon_{0}$, despite the fact that the initial strain $\epsilon_{0}$ is in the softening range. For $\epsilon_{0}=2.8 \times 10^{-3}$, by contrast, the displacement $u$ at $x=L / 4$ increases monotonically at increasing slope, and a rapid strain decrease in the middle of the rod by over $0.5 \epsilon_{0}$ is also observed [Fig. 2(b)]. This type of response is obviously unstable. At the bar ends, failure occurs.

The critical state, which is indicated by the aforementioned calculated strain histories to lie between $\epsilon_{0}=2.6 \times 10^{-3}$ and $\epsilon_{0}=2.8 \times 10^{-3}$, can be
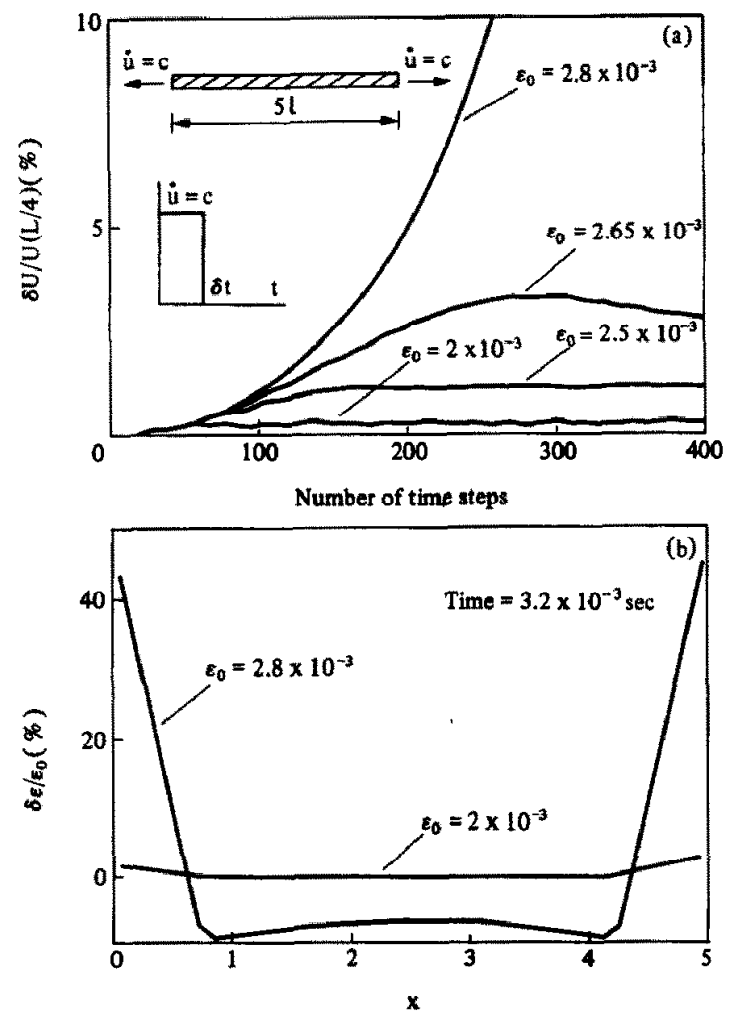

Fig. 2. Variation of displacement (a) and strain profile (b) of a bar subjected to a small dynamic perturbation of an initial strain-softening equilibrium state.
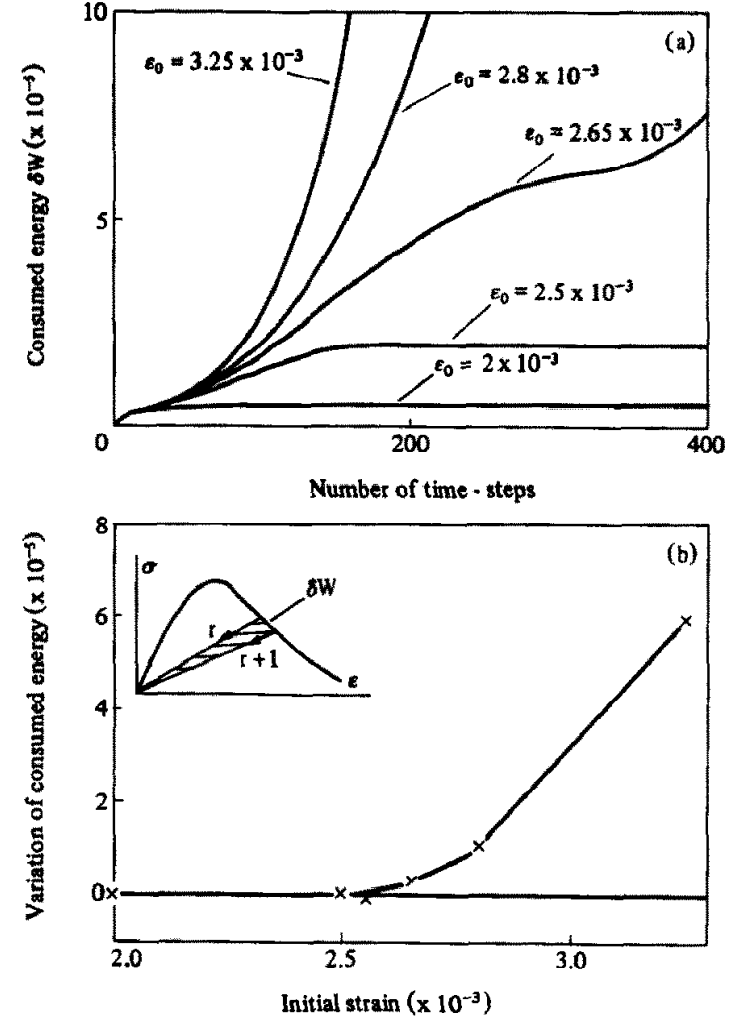

Fig. 3. Evolution of the dissipated energy consumed for a bar loaded in the softening range and subjected to a small disturbance (a); influence of the initial state on the rate of energy dissipation (b).

more sensitively detected in the evolution of the energy dissipated due to strain-softening. Energy represents a suitable measure of the overall response of the bar.

Using the same formula as in [16], we may calculate the dissipated energy $W$ from the expression

$$
\begin{aligned}
\delta W=\sum_{n=1}^{N} \frac{L_{\varepsilon}}{2}\left\{\left(\sigma_{n, r} \epsilon_{n, r}-\sigma_{n, r+1} \epsilon_{n, r+1}\right)\right. & \\
& \left.+\left(\sigma_{n r}+\sigma_{n, r+1}\right)\left(\epsilon_{n, r+1}-\epsilon_{n, r}\right)\right\},
\end{aligned}
$$

in which $n$ refers to the element number $(n=1,2,3$, $4, \ldots N)$, and subscripts $r, r+1$ refer to the beginning and the end of the $r$ th time-step. The increment of the dissipated energy density is one-half of the bracketed expression in eqn (9). It is also represented by the cross-hatched area between the unloading stress-strain diagrams from points reached at time $t_{\text {r }}$ and $t_{r+1}$ on the softening stress-strain diagram, as illustrated in Fig. 3(b).

The evolution of the dissipated energy has been calculated for the bar and is plotted in Fig. 3(a). We see that for $\epsilon_{0}<2.5 \times 10^{-3}$ the energy remains bounded and would obviously be infinitely small if the initial disturbance was infinitely small, while for $\epsilon_{0}>2.6 \times 10^{-3}$, the dissipated energy reaches large values and grows with an increasing slope. In this 
casc, of course, the energy increase is bounded by the value of the energy required to break all the material included in a zone of length approximately $l$ located at each end of the rod; for this case it appears that no matter how small the initial disturbance is, finite energy dissipation is achieved, which is an unstable situation. So the limit of dynamic stability lies between $\epsilon=2.5 \times 10^{-3}$ and $2.65 \times 10^{-3}$. Closer bounds for the stability limit could be obtained by solving further cases and using a finer subdivision of time and of the rod.

It is further useful to consider the time rate $\dot{W}$ of the energy dissipated in the bar. We plot the time rate for the final time from Fig. 3(a), i.e. for the time of 400 time-steps. Figure 3(b) shows the plot of $\dot{W}$ versus the initial strain $\epsilon_{0}$. The critical strain $\epsilon_{0}^{\text {cr }}$ lies at the point where the rising curve branches from the horizontal line. From the shape of the curve, this appears to occur betwen $\epsilon_{0}=2.5 \times 10^{-3}$ and $2.55 \times 10^{-3}$, for a bar of length $L=5 l$. For the purpose of further comparisons, we arbitrarily fix the critical state as the point at which the final energy dissipation reaches $0.01 W_{0}, W_{0}$ being the energy dissipated at the initial state of uniform strain.

Next the foregoing calculation procedure is repeated for various bar lengths $L$ in order to determine the effect of the bar length on the stability limit. The results are plotted in Fig. 4, in which we also compare the static stability limits obtained by static analysis [6], as well as the stability limit obtained by an approximate local analysis with a prescribed size $h$ of the softening zone, as originally presented [10]. Note that the curve of dynamic stability limit shown in Fig. 4 is obtained for simultaneous strain localization at both ends of the bar

The localization of strain near the ends of the bar is a typical feature of this problem $[10,17,18]$. The localization zone cannot propagate away from the ends because the existence of a strain-softening initial state precludes wave propagation. Nevertheless, the solution clearly shows that strain-softening damage spreads into a boundary region (in multidimensional situations it would be a boundary layer), the size of which is approximately equal to the characteristic length of the material $l$. A similar result has already been obtained with dynamic computations for a clamped bar [3].

From the comparison between the dynamic and static stability limits presented in Fig. 4, we note that the case where the localization does not reach the bar ends, i.e. is contained within the interior of the bar, constitutes a lower bound for dynamic stability limit.

If the bar is very long compared to $l$, however, the fact that the averaging domain protrudes beyond the boundary has a lesser influence. The difference between the localization in the interior of a very long bar and near its ends becomes small and eventually negligible, particularly in terms of the dissipated work $W$ [eqn (9)]. Therefore, the two static stability limits converge together as $L / l$ is increased. They also

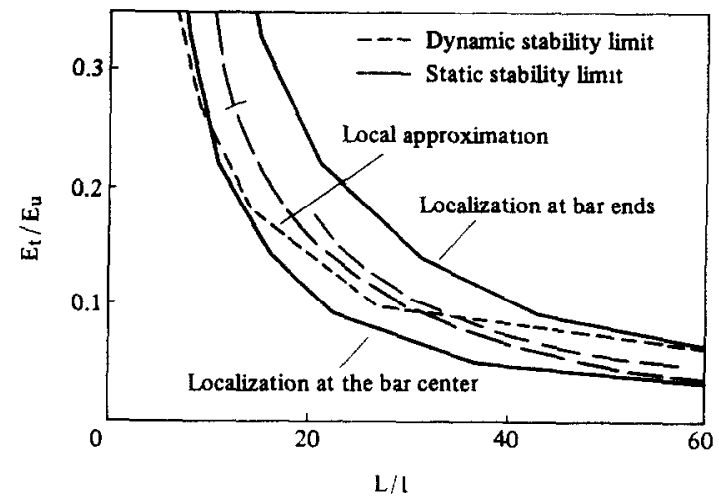

Fig. 4. Comparison of the dynamic and static stability limits

approach the strain corresponding to the peak-stress. The overall dynamic response is in this case similar to the response of one-half of the bar taken alone.

It may be noted from the calculation that the static stability limits for localization near the bar ends represent an upper bound approximation for the dynamic stability limits. In static loading, of course, the strain and damage profiles corresponding to the localization at bar ends cannot in reality develop because the localization in the interior of the bar develops earlier, i.e. at a smaller strain $\epsilon_{0}$.

One practical consequence is that, in the test of a specimen, failure should not occur, under static loading, near the loading grips but should take place in the interior of the specimen, provided the strain distribution is uniform. This appears to be confirmed by the experience with direct tensile tests of concrete which, if properly performed, normally exhibit fallure by a crack away from the grips. It is unclear, however, whether this might possibly be caused by the lateral restraint of the specimen at the grips.

\section{CONCLUSIONS}

1. The dynamic stability limit of a onedimensional bar has been calculated by applying a small disturbance at the boundary of a bar in a strain-softening equilibrium state. The nonlocal damage model, a simplified version of nonlocal continuum with local strain, is used in the analysis to avoid spurious strain localization into regions of zero volume. We find that the applied disturbance penetrates a finite distance into the bar, although it remains localized at the boundaries.

2. The stability limit is better estimated by analyzing the variation of energy consumed by damage once the disturbance has been applied. For different bar lengths, this limit was calculated in terms of critical values of damage or critical mean-tangential modulus on the strain-softening stress-strain relations. As the length of the bar increases, the size of the localization zone becomes negligible and instability occurs closer to the peak-stress. 
3. The dynamic stability condition appears to be rather close to the static limits evaluated analytically from the nonlocal formulations or simplified local model in which the size of the localization zone is specified in advance. The same size-effect on the stability limit is observed.

Acknowledgements--Partial financial support under U.S. Air Force Office of Scientific Research, Contract No. F49620-87-C-0030DEF with Northwestern University, monitored by Dr Spencer T. WU, is gratefully acknowledged. Nadine Pijaudier-Cabot deserves thanks for her valuable secretarial assistance

\section{REFERENCES}

1. K. Willam, N. Bicanic, E. Pramono and S. Sture, Composite fracture model for strain-softening computations of concrete. In: Fracture Toughness and Fracture Energy of Concrete (Edited by F. H. Wittmann), pp. 149-162. Elsevier, Amsterdam (1986).

2. Z. P. Bažant, Mechanics of distributed cracking. Appl. Mech. Rev. ASME 39, 675-705 (1986).

3. G. Pijaudier-Cabot and Z. P. Bazant, Nonlocal damage theory. J. Engng Mech. Div., ASCE (in press). See also Report No. 86-8/428n, Northwestern University, Evanston, IL (1986).

4. P. Ladevèze, On an anisotropic damage theory (in French). Internal Report, No. 34, Laboratoire de Mécanique et Technologie, France (1983).

5. J. Mazars and G. Pijaudier-Cabot, Continuum damage theory: application to concrete. Internal Report No. 71, Laboratoire de Mécanique et Technologie, Cachan, France (1986).

6. Z, P. Bažant and G. Pijaudier-Cabot, Nonlocal damage: continuum model and localization instability. Report No. 87-2/428n-I, Center of Concrete and Geomaterials, Northwestern University, Evanston, IL (1987).

7. J. Lemaitre and J. L. Chaboche, Mécanique des Matériaux Solides. Dunod-Bordas, Paris (1985).
8. A. Zaborski, An isotropic damage model for concrete (in French). Internal Report No. 55, Laboratoire de Mécanique et Technologie, ENSET, Cachan, France (1985).

9. Z. P. Bažant, F. B. Lin and G. Pijaudier-Cabot, Yield limit degradation: nonlocal continuum with local strains. Preprints, Int. Conf. on Computational Plasticity, held in Barcelona (Edited by E. Onate, R. Owen and E. Hinton). University of Wales, Swansea (1987).

10. Z. P. Bažant, Instability, ductility, and size effect in strain-softening concrete. J. Engng Mech. Div., ASCE 102, 331-344 (1976); discussions 103, 357-358, 775-177; 104, 501-502 (based on Struct. Engng Report No. 74-8/640, Northwestern University, IL, August 1974)

11. Z. P. Bažant, G. Pijaudier-Cabot and J. Pan, Ductility, snapback, size effect and redistribution in softening beams of frames. Report No. 86-7/428d, Center of Concrete and Geomaterials, Northwestern University, Evanston, IL (1986), see also J. Struct. Engng, ASCE (in press).

12. Z. P. Bažant and A. Zubelewicz, Strain-softening bar and beam; exact nonlocal solution. Report, Center for Concrete and Geomaterials, Northwestern University, Evanston, IL (1986).

13. N. S. Ottosen, Thermodynamic consequences of strainsoftening in tension. J. Engng. Mech. Div., ASCE 112, 1152-1164 (1986).

14. Z. P. Bažant and T. B. Belytschko, Wave propagation in a strain-softening bar: exact solution. J. Engng Mech. Div., ASCE 111, 381-389 (1985).

15. F. H. Wu and L. B. Freund, Deformation trapping due to thermoplastic instability in one-dimensional wave propagation. J. Mech. Phys. Solids 21, 119 (1985).

16. Z. P. Bažant, T. B. Belytschko and T. P. Chang, Continuum theory for strain-softening. J. Engng Mech. Div., ASCE 110, 1666-1692 (1984).

17. Z. P. Bažant and T. B. Belytschko, Strain-softening continuum damage: localization and size effect. Preprints, Int. Conf. on Constitutive Laws for Engineering Materials, Vol. 1 (Edited by C. S. Desai et al.), pp. 11-33. Elsevier, New York (1987).

18. Hadamard, Leçons Sur la Propagation des Ondes, Ch. VI. Hermann et Cie, Paris (1903). 